\title{
Análisis antropológico físico del cementerio Mocha 2 (norte de Chile, I Región)
}

Vivien STANDEN ${ }^{1}$ y Julio Sanhueza ${ }^{2}$

\section{Introducción}

Este informe corresponde al estudio de los restos óseos humanos de un cementerio prehistórico tardío (ca. 1200-1500 DC). Se encuentra ubicado en la banda norte del curso superior de la quebrada de Tarapacá, sector Mocha, a una distancia aproximada de $2 \mathrm{~km}$ al sur del pueblo del mismo nombre y a los pies de las ruinas de un pucara. El sector dista aproximadamente $140 \mathrm{~km}$ de la costa y tiene una altura de $2400 \mathrm{~m} . \mathrm{snm}$, caracterizándose por recursos hídricos regulares, con una producción agrícola que pudo permitir la estabilidad socioeconómica de las poblaciones del pasado.

Los registros culturales, que están en proceso de estudio, indicarían la presencia de un desarrollo local, en contacto con poblaciones altiplánicas tardías portadoras de cerámica del horizonte Negro sobre Rojo (Sanhueza Ms-a). En cuanto a las ofrendas funerarias se registran ceramios decorados (botellones, jarros de agua) y pulidos o alisados (jarros, pucos, platos, ollas y otros); vasos tallados y cajitas de madera; elementos de industria textil (aunque los textiles son escasos por mala preservación), además, hay huesos de camélidos, pintura ocre y verde en calabazas; cestería en espiral y collares en cuentas de piedras (Sanhueza Ms-a).

\section{Características del cementerio}

En relación a la posición y distribución de las tumbas en el talud inclinado, éstas se encuentran en distintos niveles de profundidad (algunas a $30 \mathrm{~cm} \mathrm{y}$ otras hasta $1 \mathrm{~m}$ ), por una parte a causa de los escurrimientos de barro por lluvias, que hicieron crecer la capa superficial de detritus y tierra sobre algunas tumbas. Los enterramientos se hicieron generalmente en fosos circulares y sus rellenos corresponden a material del mismo talud, son de carácter individual,

1 Instituto de Antropología, Universidad de Tarapacá, Arica, CHILE.

2 Universidad Arturo Prat, Iquique, CHILE. aunque hay también enterramientos secundarios. Los cuerpos se ubican en posición semisentada, con las extremidades inferiores flectadas. Algunos conglomerados de funebria han sido cubiertos por camadas de fibra vegetal y postes demarcatorios, sellados por lajas y rodados. Los neonatos y lactantes se encuentran depositados individualmente en ceramios (Sanhueza Ms-b).

\section{Estado del material y método}

El cementerio sufrió fuertes perturbaciones, por lo que la mayor parte de los esqueletos no se encuentran en óptimas condiciones, presentándose algunos huesos muy frágiles y sin ningún tipo de tejido blando. Hay cuerpos incompletos, y algunos individuos bien preservados. Los esqueletos rescatados alcanzaron a 21 individuos, quedando in situ una parte importante del cementerio.

Para determinar la edad y sexo de la población se utilizaron los parámetros propuestos por Genovés (1962). En el análisis métrico nos basamos en Hrdlicka (1939), Comas (1966), Bass (1972). Aplicamos la fórmula de Genovés (en Ubelaker 1978) para determinar la estatura, que está basada en muestras mesoamericanas.

\section{Resultados}

Se presentan los resultados métricos de los cráneos evaluados, además de un cuadro de distribución de edad y sexo de una parcialidad de la población, ya que el cementerio no está excavado en su totalidad, esperando a futuro continuar los trabajos de campo (Tablas 1, 2 y 4).

\section{Tipo físico}

Los cráneos que presentaron óptimas condiciones para evaluar las diferentes medidas e índices alcanzaron una reducida muestra (dos femeninos, tres masculinos). Manifiestan una morfología mesobraquicéfala, ancho intermedio y altura mediana, 
correspondiendo a la serie femenina. Los cráneos masculinos presentaban deformación artificial, por lo que no se evaluaron (Tabla 2). Respecto a los caracteres faciales se evalúan dos femeninos $\mathrm{y}$ tres masculinos, son de caras mesenas (ancho intermedio), narices camerrinas (anchas), órbitas hipsiconcas (altas), paladar mesobraquiestafilino (tendencia a la anchura), no existiendo un marcado dimorfismo sexual en estos aspectos. Esta morfología no difiere de otras poblaciones tardías del interior y/o tierras altas, como es el caso de los individuos del cementerio Usamaya 1 de Isluga (Sanhueza 1981) que tienen similares caracteres y cuya ubicación geográfica no es tan distante del sector en estudio. Por otra parte, 11 individuos excavados por Ryden (1947) y estudiados por Hjostjo y Lindh (1947) en varios cementerios del altiplano boliviano y con patrones similares al cementerio Usamaya 1 no presentan tampoco grandes diferencias faciales (mésenos, hipsiconcos), no pudiendo compararse las bóvedas por su deformación cultural. Obtenidos en una cuenca alta, los esqueletos incaicos de El Tojo valle de Collacagua (Ericksen 1962) hacen apreciar un notable distanciamiento no sólo con Mocha 2, sino con casi todas las poblaciones tardías del interior que han sido descritas, por su morfología notoriamente diferente y su estatura. Por último, en la población de Toconce o Likan (Quevedo 1979) (complejo cultural altiplánico en la cuenca alta del río Salado), observamos cierta variación en cuanto a la altura del cráneo (son más altos) y la forma del paladar (angosto y alto) pero no así en otros parámetros donde tienen cercanía a Mocha 2.

\section{Estimaciones sobre estatura}

En relación al cálculo de estatura determinamos un promedio de $157.118 \mathrm{~cm}$ para los hombres, siendo las mujeres de estatura más baja, con $151.173 \mathrm{~cm}$. Estos resultados concuerdan con otras poblaciones del área, a excepción de la población El Tojo (Ericksen 1962) que son notoriamente más altos (Tabla 3 ).

\section{Patologías y observaciones óseas}

Las patologías que pudimos identificar corresponden a las de carácter degenerativo y algunos procesos infecciosos. Es así como en 10 individuos adultos analizados (hay que destacar que no todos los cuerpos están completos, lo que dificulta el análisis), solo tres esqueletos manifiestan espondilosis incipiente a nivel de columna lumbar y procesos de uncoartrosis a nivel de cuello en las vértebras cervicales, dos cuerpos corresponden a hombres y uno a mujer. Esto nos sugiere una baja frecuencia de este tipo de patologías.

Se detectó un caso de periostitis en una extremidad inferior de radio izquierdo que pertenece a un adulto masculino.

Patologías de carácter traumático observamos solo en un individuo adulto masculino (T-10), que exhibe un gran callo óseo producto de una fractura a nivel del tercio distal de la tibia y peroné derecho, donde se aprecia una desalineación del eje del hueso, que debió haber traído una alteración anátomo-funcional del pie, ya que manifiesta una exostosis plantar a nivel de la articulación metatarso-falángica y calcáneoastragalina en su carilla anterior. Esta extremidad quedó acortada en $4 \mathrm{~cm}$ con respecto a su homóloga. En el acromion de la escápula izquierda se observa la formación de una carilla articular, allí se produjo una fractura que no tuvo consolidación, formándose una seudoarticulación. La cuarta vértebra cervical a nivel de su cuerpo ha perdido considerablemente su altura, se suma a esto un aplastamiento en forma de cuña con $1 \mathrm{~cm}$ de diferencia respecto a su borde posterior. Este individuo pudo haber estado sometido a condiciones de violencia en relación a su medio o con otro individuo.

Respecto a anomalías de carácter congénito se registró una fusión ósea a nivel de la articulación tibio-peronea inferior (T-9), la que se encuentra mal preservada; corresponde a un sujeto adulto del cual no se pudo determinar el sexo en los escasos huesos presentes. Llama también la atención una fusión ósea a nivel de la articulación sacroilíaca en su parte anterior; hemos evaluado esta situación como un núcleo de osificación secundario de carácter congénito, ya que su morfología no corresponde a procesos degenerativos o exostosis ósea. Como rasgo importante destacamos que esto se observó en dos individuos adultos, ambos de sexo femenino: en un caso es bilateral (T-5a) (presentándose en ambas articulaciones) y en el otro es unilateral (sólo en lado izquierdo). Esta situación no ha sido descrita con anterioridad en otras poblaciones prehistóricas del área, al menos en la bibliografía revisada.

\section{Observaciones dentarias}

Hay un alto porcentaje de piezas dentarias perdidas en vida, incluyendo individuos con total anodoncia, 


\begin{tabular}{|c|c|c|c|c|c|c|c|}
\hline \multirow{2}{*}{ Medidas (mm) } & \multicolumn{4}{|c|}{ Masculinos } & \multicolumn{3}{|c|}{ Femeninos } \\
\hline & $\mathbf{T}_{10}$ & $\mathbf{T}_{5 \mathrm{~h}}$ & $\mathbf{T}_{13}$ & Prom. & $\mathbf{T}_{7}$ & $\mathrm{~T}_{8}$ & Prom. \\
\hline D. bas breg. & - & - & - & - & 125 & 127 & 126 \\
\hline D. glab. opist. & - & - & - & - & 169.5 & 165 & 16.72 \\
\hline D. eu. eu. & - & - & - & - & 133 & 133 & 133 \\
\hline Alt. nas. gna. & 11.3 & 10.5 & 10.5 & 10.76 & 107 & 9.2 & 9.95 \\
\hline Alt. nas. prost. & 6.5 & 6.3 & 6.3 & 6.36 & 6.5 & 6.4 & 6.45 \\
\hline D. front. min. & - & - & 8.5 & - & 8.2 & 8.28 & 8.24 \\
\hline D. bizig. & 12.1 & 13.4 & - & 12.75 & 121 & 12.11 & 12.1 \\
\hline Alt. nariz & 4.3 & 4.75 & 4.8 & 4.61 & 43 & 48 & 4.55 \\
\hline Ancho nariz & 2.4 & 2.8 & 2.5 & 2.56 & 2.4 & 2.35 & 2.37 \\
\hline Alt. órbita & 3.35 & 3.75 & 3.85 & 3.65 & 3.3 & 3.59 & 3.44 \\
\hline Dac. ectocon. & 3.5 & 3.55 & 3.65 & 3.56 & 3.5 & 3.6 & 3.55 \\
\hline D. bas. prost. & 9.4 & 8.5 & - & 8.95 & 9.4 & 8.85 & 9.12 \\
\hline D. bimast. & 11.1 & 13.5 & - & 12.3 & 11.1 & 10.9 & 11.0 \\
\hline Long. alveolar & 5.4 & 5.2 & 5.3 & 5.3 & 5.4 & 5.0 & 5.2 \\
\hline Ancho alveolar & 59 & 5.5 & 6.7 & 6.0 & 5.9 & 5.0 & 5.45 \\
\hline Long. palatina & 4.3 & 4.3 & 4.1 & 4.23 & 4.3 & 4.65 & 4.47 \\
\hline Ancho palatino & 3.6 & 3.9 & 4.3 & 3.93 & 3.6 & 3.8 & 3.7 \\
\hline D. bigonial & 8.9 & 9.75 & 10.4 & 9.68 & 8.9 & 8.55 & 8.7 \\
\hline D. bicond. & 11.15 & 12.5 & 13.3 & 12.31 & 11.15 & 11.7 & 11.42 \\
\hline Alt. sinf. ment. & 3.0 & 2.8 & 2.6 & 2.8 & 3.0 & 2.65 & 2.82 \\
\hline Alt. rama & 5.9 & 5.1 & 6.5 & 5.83 & 5.9 & 5.7 & 5.8 \\
\hline Ancho rama & 3.25 & 2.3 & 3.4 & 2.98 & 3.25 & 3.2 & 3.22 \\
\hline Long. cond. sínfis. & 12.0 & 11.6 & 12.4 & 12.0 & 11.05 & 10.9 & 11.48 \\
\hline
\end{tabular}

Tabla 1. Craneometría de Mocha 2.

\begin{tabular}{|l|c|c|c|c|c|c|c|}
\hline \multirow{2}{*}{ Indices } & \multicolumn{4}{c|}{ Masculinos } & \multicolumn{3}{c|}{ Femeninos } \\
\cline { 2 - 9 } & $\mathbf{T}_{\mathbf{1 0}}$ & $\mathbf{T}_{\mathbf{5 h}}$ & $\mathbf{T}_{\mathbf{1 3}}$ & Prom. & $\mathbf{T}_{\mathbf{7}}$ & $\mathbf{T}_{\mathbf{8}}$ & Prom. $^{*}$ \\
\hline Cefal. horizontal & - & - & - & - & 78.46 & 80.60 & 79.53 \\
\hline Mixto alt. craneal & - & - & - & - & 82.67 & 85.23 & 83.95 \\
\hline Vértigo transc. c/bas. & - & - & - & - & 93.98 & 95.48 & 94.73 \\
\hline Vértigo long. c/bas. & - & - & - & - & 73.74 & 76.96 & 75.35 \\
\hline Facial superior & 53.71 & 47.01 & - & 50.36 & 53.71 & 52.84 & 53.27 \\
\hline Facial total & 93.38 & 78.35 & - & 85.86 & 88.42 & 75.97 & 82.19 \\
\hline Nasal & 55.81 & 58.94 & 52.8 & 55.61 & 55.81 & 48.95 & 52.38 \\
\hline Orbitario & 94.28 & 100.0 & 102.7 & 98.99 & 94.28 & 99.72 & 97.70 \\
\hline Palatino & 83.72 & 90.69 & 104.87 & 93.09 & 83.72 & 8.72 & 82.72 \\
\hline Alveolar & 109.25 & 105.76 & 126.41 & 113.8 & 109.25 & 100,0 & 104,62 \\
\hline Frontoparietal & - & - & - & - & 61.65 & 62.25 & 61.95 \\
\hline Mandíbula & 107.62 & 92.8 & 93.23 & 97.88 & 99.10 & 93.16 & 96.13 \\
\hline
\end{tabular}

Tabla 2. Indices craneométricos para Mocha 2. 


\begin{tabular}{|c|c|c|c|c|c|c|}
\hline & Mascu & & & & & \\
\hline \multirow[t]{5}{*}{ Fémur: } & $\mathrm{t}-1$ & $=$ & 39.1 & - 154.745 & $+\quad(158.162$ & - 151.328$)$ \\
\hline & $t-10$ & $=$ & 39.45 & $-\quad 155.536$ & $+\quad(158.953$ & $-\quad 152.119)$ \\
\hline & $\mathrm{t}-5 \mathrm{~h}$ & $=$ & 41.9 & - $\quad 161.073$ & $+\quad(164.490$ & $-\quad 157.656)$ \\
\hline & & & Prom. & 157.118 & (160.535 & - 153.701$)$ \\
\hline & Femen & & & & & \\
\hline \multirow[t]{5}{*}{ Fémur: } & $t-2$ & $=$ & 40.2 & -153.86 & $+\quad(157.676$ & $-\quad 150.044)$ \\
\hline & $\mathrm{t}-5 \mathrm{a}$ & $=$ & 40.2 & -153.86 & $+\quad(157.676$ & $-\quad 150.044)$ \\
\hline & $\mathrm{t}-7$ & $=$ & 38.45 & - 149.328 & $+\quad(153.144$ & $-\quad 145.512)$ \\
\hline & $t-8$ & $=$ & 37.8 & $-\quad 147.644$ & $+\quad(151.460$ & $-\quad 143.828)$ \\
\hline & & & Prom. & 151.173 & 154.989 & 147.357 \\
\hline
\end{tabular}

(*) Las medidas se encuentran expresadas en centímetros. Las fórmulas utilizadas son las de Santiago Genovés (1967) en Ubelaker (1978: 45):

Masculino $\quad 2.26$ x Long. fémur $+66.379 \pm 3.418$

Femenino $\quad 2.59 \times$ Long. fémur $+49.742 \pm 3.816$

No se restaron los $2.5 \mathrm{~cm}$ que sostiene la fórmula.

Tabla 3. Estatura (*).

\begin{tabular}{|c|c|c|c|c|c|c|c|c|}
\hline \multicolumn{2}{|c|}{$\begin{array}{l}\text { Neonatos } \\
\text { lactantes }\end{array}$} & \multicolumn{2}{|c|}{$\begin{array}{c}\text { Niños } \\
3-12\end{array}$} & \multirow[t]{2}{*}{$\begin{array}{c}\text { Subadultos } \\
13-18\end{array}$} & \multicolumn{2}{|c|}{$\begin{array}{c}\text { Adulto joven } \\
19-25\end{array}$} & \multicolumn{2}{|c|}{$\begin{array}{c}\text { Adultos edad } \\
\text { indeterminada } \\
>26\end{array}$} \\
\hline & I & 3 & I & & 2 & $\mathrm{~F}$ & 1 & $\mathrm{M}$ \\
\hline & I & & I & & 7 & F & 4 & I \\
\hline & I & $5 f$ & I & & & & $5 \mathrm{a}$ & $\mathrm{F}$ \\
\hline & I & & I & & & & $5 \mathrm{~h}$ & M? \\
\hline & I & & & & & & 8 & $\mathrm{~F}$ \\
\hline \multirow{3}{*}{\multicolumn{2}{|c|}{14}} & & & & & & 9 & I \\
\hline & & & & & & & 10 & $\mathrm{M}$ \\
\hline & & & & & & & 13 & $\mathrm{M}$ \\
\hline
\end{tabular}

Simbología

$\begin{array}{llll}\mathrm{N}^{\circ} & : & \text { número del esqueleto } \\ \text { Sexo } & \text { M } & : & \text { masculino } \\ & \text { F } & : & \text { femenino } \\ & \text { I } & : & \text { indeterminado } \\ & \text { M? } & : & \text { masculino probable } \\ & \text { F? } & : & \text { femenino probable }\end{array}$

Tabla 4. Distribución de edad y sexo de la población Mocha 2.

lo cual indica que los procesos infecciosos debieron ser frecuentes y graves. Si se compara esta población agrícola con otra precerámica, por ejemplo Tiliviche 2 (Standen 1981), vemos una notable diferencia, ya que en esta última el número de piezas dentarias perdido es mínimo al igual que la incidencia de caries, debido principalmente a la diferenciación de la dieta.
En cuanto al grado abrasivo, éste es moderado, a excepción de un individuo adulto masculino donde es marcado y compromete con mayor frecuencia a los incisivos, predominando el tipo de abrasión plana. Esto nos indica que el tipo de dieta debió ser elaborada y sometida a procesos de cocción (existen ollas con restos de hollín en su base y tiestos con harinas). 
Se observó un diente erupcionado en la línea media de la sínfisis mentoniana de un individuo femenino adulto.

En los escasos incisivos presentes se manifiesta el típico "diente en pala", característico de las poblaciones mongoloides.

\section{Deformación craneana intencional}

De una muestra de seis cráneos, solo en dos se observa deformación intencional, no siendo común este rasgo. La deformación tiene cierta similitud en ambos cráneos que además corresponden al sexo masculino.

El individuo de la tumba T-10, que presentó en la excavación una especie de casco en barritas de madera, mostró una depresión a modo de incipiente cintura en el borde anterior de ambos parietales, pero sin compromiso de la zona bregmática. El aparato deformador debió implicar elementos flexibles con rellenos a modo de almohadillas. El otro cráneo (T-5 h) manifiesta además de los caracteres anteriores una suave depresión supramastoidea con compromiso de la articulación temporo-occipital. Desgraciadamente no se registró ningún aparato deformador durante la excavación, pero ambas deformaciones se aproximan a la norma circular, ya descrita para las poblaciones del norte de Chile (Munizaga 1964; Soto 1974).

\section{Conclusiones}

Hasta ahora, con sólo parte de la población inhumada en el cementerio tardío de Mocha 2, podemos concluir que el tipo físico que exhiben es: cráneos mesobraquicéfalos, bóvedas de mediana altura y tamaño con un ancho intermedio en la serie femenina ya que los cráneos masculinos se encuentran deformados. Tienen caras mesenas (ancho mediano), narices camerrinas (anchas), órbitas hipsiconcas (altas), paladar mesobraquiestafilino (tendencia a ser anchos), sin mostrar un notable dimorfismo sexual.

En lo dentario se observó a grandes rasgos la presencia del sistema de neutroclusión, habiendo existido graves procesos infecciosos. La abrasión no es muy acentuada, predominando la de tipo plana.

Se deformaban el cráneo en una forma que asemeja al tipo circular.

En general son individuos no demasiado robustos, con una estatura baja con un promedio de 151.173 $\mathrm{cm}$ para las mujeres y $157.118 \mathrm{~cm}$ para los hombres. En patologías óseas, se observan sólo algunos procesos degenerativos (espondiloartresis, uncoartosis) en columna vertebral y traumatismos (fracturas en huesos largos) por violencia.

La obtención de nuevos datos producto de excavaciones futuras permitirá completar los planteamientos expuestos.

\section{REFERENCIAS CITADAS}

BASS, W., 1972. Human osteology: A laboratory and field manual of the human skeleton. University of Missouri, Columbia, Missouri.

COMAS, J., 1966. Manual de antropología física. Universidad Autónoma de México, México D. F.

ERICKSEN, M. F., 1962. Restos óseos encontrados en El Tojo. Revista Universitaria XLVII: 143-149.

GENOVES, S., 1962. Introducción al diagnóstico de la edad y sexo en restos óseos prehistóricos. Universidad Autónoma de México, México D. F.

HJORTSJO, C. y J. LINDH, 1947. Anthropological investigation of cranial and skeleton material. En Archaeological researches in the Highlands of Bolivia, S. Rydén (Ed.). Gotemburgo.
HRDLICKA, A., 1939. Practical anthropometry. Widtar Institute of Anatomy and Biology of Philadelphia, Filadelfia.

MUNIZAGA, J., 1980. Esquema de la antropología física del norte de Chile. Chungara 6: 124-136.

QUEVEDO, S., 1979. Comunicación personal al Simposio: Período Tardío en el Area Centro Sur Andina. Actas del VIII Congreso de Arqueología Chilena (Valdivia). Ediciones Kultrún, Santiago.

SANHUEZA, J., Ms-a. Valles altos y altiplano en el período: Problemas y nuevos aportes (Provincia de Iquique, Región de Tarapacá). Instituto Profesional de Iquique. Iquique.

Ms-b. Apuntes de campo del cementerio Mocha 2. 
— 1981. Estudio de los restos óseos del cementerio Usamaya 1, altiplano de Isluga. I Región. Documentos de Trabajo 8.

SOTO, P., 1974. Análisis antropológico físico de restos humanos correspondientes a Chinchorro, El Laucho y Alto Ramírez. Chungara 3: 85-93.
STANDEN, V., 1981. Análisis antropológico físico de una población prehistórica del norte de Chile. Tesis para optar al Título de Kinesiólogo. Universidad del Norte, Antofagasta.

UBELAKER, D., 1978. Human skeletal remains, excavation, analysis, interpretation. Aldine Manuals on Archaeology. Smithsonian Institution, Chicago. 